\title{
Make it two: A case report of dual sequential external defibrillation
}

\author{
Colin R. Bell, $\mathrm{MD}^{*}{ }^{\dagger}$; Adam Szulewski, MD, $\mathrm{MHPE}^{\dagger}$; Steven C. Brooks, MD, MHSc ${ }^{\dagger}$
}

\section{ABSTRACT}

Dual sequential external defibrillation (DSED) is the process of near simultaneous discharge of two defibrillators with differing pad placement to terminate refractory arrhythmias. Previously used in the electrophysiology suite, this technique has recently been used in the emergency department and prehospital setting for out-of-hospital cardiac arrest (OHCA). We present a case of successful DSED in the emergency department with neurologically intact survival to hospital discharge after refractory ventricular fibrillation (RVF) and review the putative mechanisms of action of this technique.

\section{RÉSUMÉ}

La défibrillation externe séquentielle double est une technique de décharge quasi simultanée de deux défibrillateurs dont les plaques sont situées en des points différents du thorax pour mettre fin à des troubles réfractaires du rythme. Appliquée auparavant au service d'électrophysiologie, la technique est utilisée depuis peu au service des urgences et en milieu préhospitalier pour les arrêts cardiaques qui surviennent dans la collectivité. Sera exposé ici un cas réussi de défibrillation externe séquentielle double au service des urgences, qui a permis une survie intacte, sans séquelles neurologiques, jusqu'au moment du congé de l'hôpital, après un épisode de fibrillation ventriculaire réfractaire; suivront des hypothèses quant aux mécanismes d'action possibles de cette technique.

Keywords: dual sequential external defibrillation, refractory ventricular fibrillation, ventricular fibrillation

\section{INTRODUCTION}

More than 45,000 Canadians suffer OHCA annually, with only $8.4 \%$ surviving to hospital discharge. ${ }^{1,2}$ Current treatment strategies for OHCA focus on high quality cardiopulmonary resuscitation (CPR), early rhythm identification, and defibrillation for shockable rhythms such as ventricular fibrillation (VF) or pulseless ventricular tachycardia $(\mathrm{pVT}){ }^{3}$
Unfortunately, a proportion of patients with VF does not respond to standard therapy and succumb to VF, despite multiple defibrillation attempts. The definition of shock RVF varies in published literature. In the recent Resuscitation Outcomes Consortium - Amiodarone, Lidocaine, or Placebo Study (ROC-ALPS), of 37,889 patients with non-traumatic OHCA considered for enrolment, $18.6 \%$ had shock RVF or pVT, defined as refractory to at least one shock by emergency medical services (EMS) personnel. ${ }^{4}$ Sakai et al. found the incidence of $\mathrm{VF}$ that was resistant to at least one shock by EMS has an incidence of 0.5-0.6 cases per 100,000. ${ }^{5}$ Others have defined RVF as VF that has not responded to five defibrillation attempts at standard energy with ongoing high quality advanced cardiovascular life support (ACLS) care. ${ }^{6-8}$ Patients with RVF have dismal outcomes because there are no well-established treatment options available. ${ }^{5,7}$ Recently, a small study of esmolol administration during VF refractory to at least three shocks demonstrated some success increasing the rate of return of spontaneous circulation (ROSC); however, the improvement in the rate of neurologically intact survival was not statistically significant. ${ }^{9}$ Some jurisdictions use mechanical CPR, extracorporeal membrane oxygenation (ECMO) assisted resuscitation, or early percutaneous coronary intervention (PCI) during $\mathrm{VF}$, but these interventions are not routine in most emergency departments and not readily achievable outside of large urban centres.

DSED involves the use of two defibrillators and two sets of defibrillator pads (Figure 1). As soon as the two sets of pads are applied to the patient, both defibrillators are discharged nearly simultaneously. DSED has been described and studied in the electrophysiology suite for

From the *Department of Emergency Medicine, Denver Health, Denver, CO; and tDepartment of Emergency Medicine, Queen's University, Kingston, ON.

Correspondence to: Dr. Adam Szulewski, Department of Emergency Medicine, Queen's University, 76 Stuart St., Kingston, ON K7L 2V7; Email: aszulewski@qmed.ca 
A

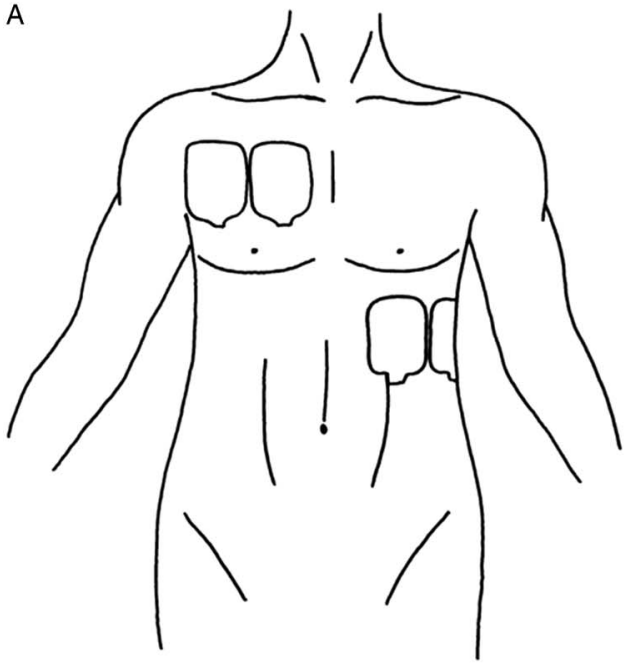

B

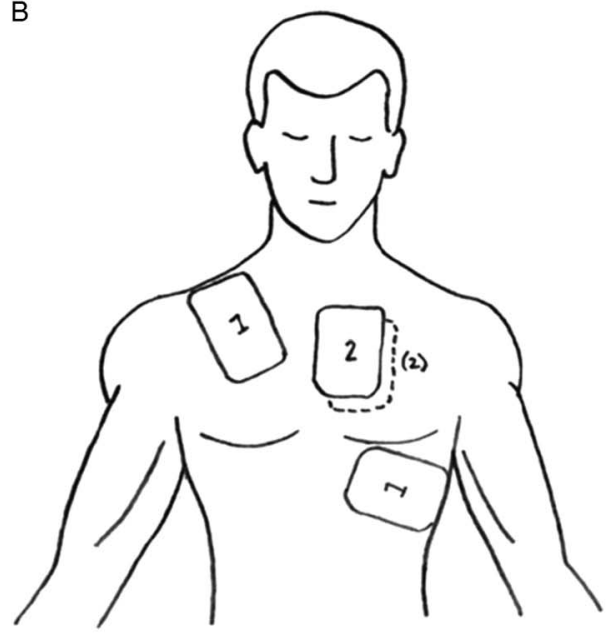

Figure 1. A) Pad placement. B) Defibrillator pad placement. ( $A$ - Reproduced with permission from Lybeck A, Moy H, Tan D. Double sequential defibrillation for refractory ventricular fibrillation: a case report. Prehosp Emerg Care 2015;19[4]:554-7; $B$ - Reproduced with permission from Leacock B. Double simultaneous defibrillators for refractory ventricular fibrillation. J Emerg Med 2014;46[4]:472-4.)

the treatment of refractory arrhythmias ${ }^{10-12}$ but has not translated into mainstream medical care for OHCA.

We present a case of successful use of DSED in our Canadian emergency department for a patient with RVF. Consent was obtained from the patient to publish the details of his case in accordance with guidelines set out by the Queen's University Health Sciences Research Ethics Board (File number: 6018904).

\section{CASE}

A 53-year-old male with a previous history of osteoarthritis, psoriasis, and hypertension (treated with ramipril) developed chest pain and nausea while cleaning out his garage. A 911 call was made, and a primary care paramedic crew arrived at 1136 hours to find an overweight (body mass index [BMI] 28.4), pale, and diaphoretic patient who was complaining of ongoing 4/10 chest heaviness radiating to his back and left arm, with a pulse of 106 and an indeterminate blood pressure. The patient reported a similar episode of chest pain 4 days prior that had resolved spontaneously. Acetylsalicylic acid (ASA) $160 \mathrm{mg}$ was administered, but, prior to initiation of transport at 1139 hours, the patient developed VF. He was immediately defibrillated using a Zoll $\mathrm{X}$ series defibrillator and a biphasic shock of 200 joules $(J)$. The defibrillator pads were placed in the standard anterolateral configuration. He regained consciousness within seconds, with a subsequent pulse of 84 and blood pressure of 110/66 mm Hg.
An on-scene 12-lead electrocardiogram (ECG) demonstrated sinus rhythm and $4 \mathrm{~mm}$ of ST-segment elevation in V1-V3 (Figure 2, A). The local ST-elevation myocardial infarction protocol was activated at 1152 hours, and transport to the regional PCI centre commenced.

At 1159, while still en route, a second episode of $\mathrm{VF}$ occurred (see Figure 2, B), this time refractory to two defibrillation attempts at $200 \mathrm{~J}$, chest compression, and bag-valve-mask (BVM) ventilation by paramedics.

On arrival in the emergency department at 1205 hours, the patient had no vital signs and continued to be in VF. A third attempt at defibrillation with $200 \mathrm{~J}$ was made prior to transfer onto the emergency department stretcher and was unsuccessful. Intravenous access was obtained and tenecteplase $50 \mathrm{mg}$, amiodarone $300 \mathrm{mg}$, and epinephrine $1 \mathrm{mg}$ intravenous were administered to maximize the probability of myocardial reperfusion given the presumed cardiac etiology of his cardiac arrest. A fourth defibrillation attempt using a biphasic Lifepak 20 at $200 \mathrm{~J}$ with the traditional anterolateral pad placement was performed shortly thereafter and was also unsuccessful. The patient was intubated with paralytic assistance during ongoing CPR using direct laryngoscopy, and the decision was made to attempt DSED. A second Lifepak 15 defibrillator was applied in an anteroposterior position and two providers, each operating one defibrillator, delivered near simultaneous $200 \mathrm{~J}$ biphasic shocks at 1216 hours. The patient was successfully defibrillated into a narrow complex bradycardia and had a palpable pulse at 1218 hours. 
A
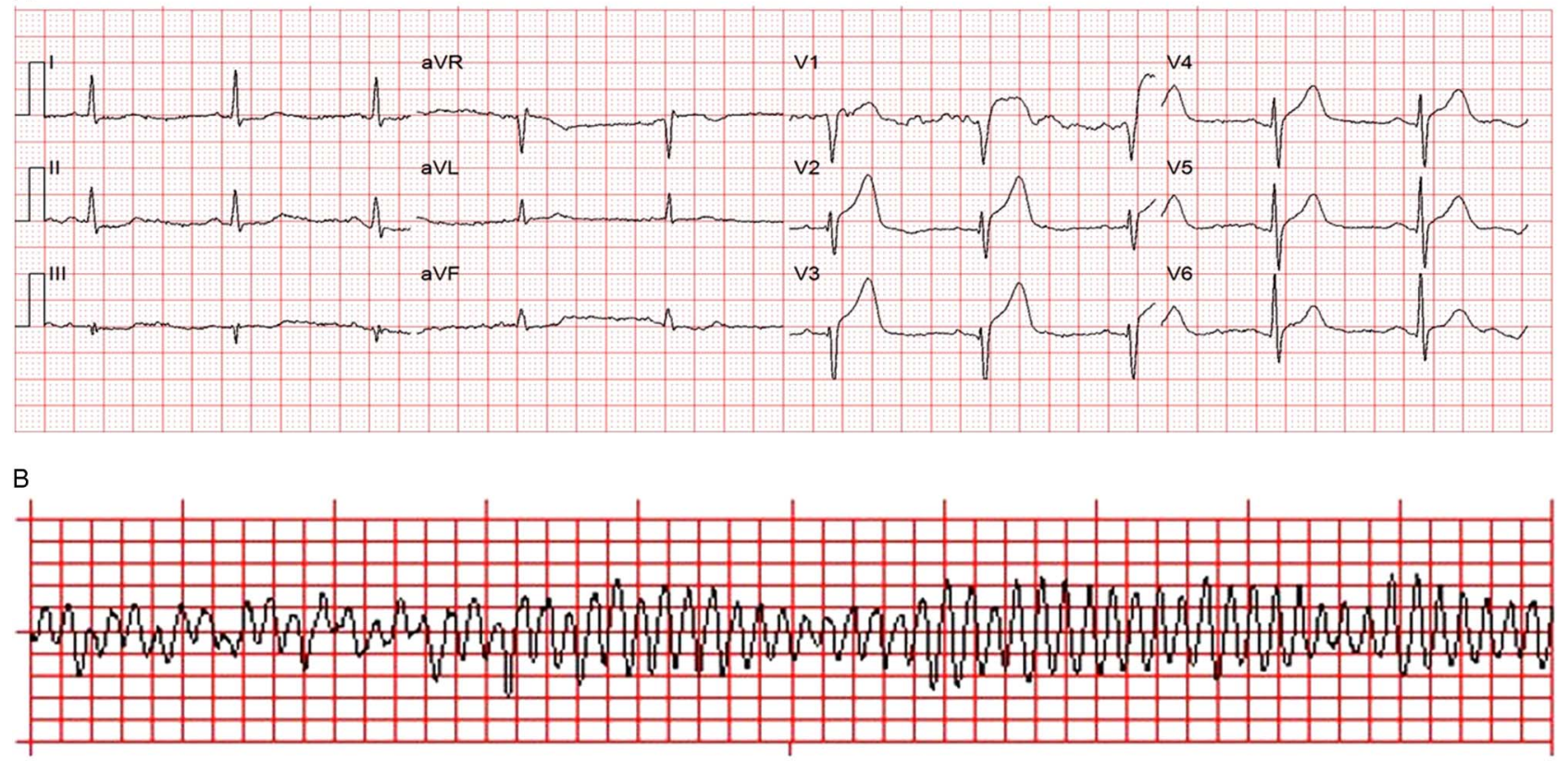

C

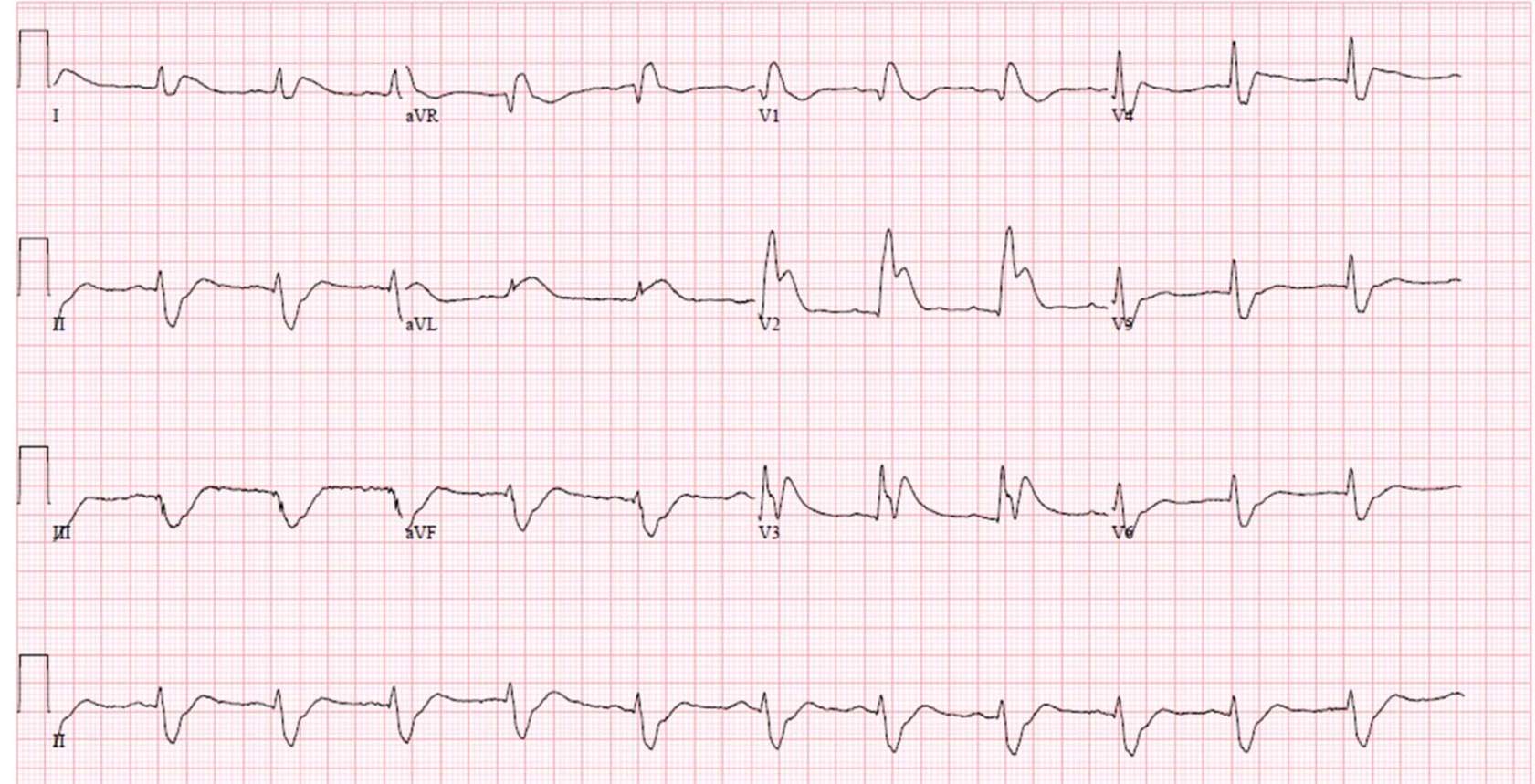

Figure 2. A) Prehospital 12-lead ECG. B) Prehospital rhythm strip. C) Emergency department 12-lead ECG after successful DSED.

Within 60 seconds, VF recurred. A second DSED attempt at $200 \mathrm{~J}$ from each defibrillator at 1222 hours successfully defibrillated the patient back to sinus rhythm with a heart rate of 53/minute, blood pressure of 115/97 mm Hg, and Glasgow Coma Scale of 3T. Emergency department 12-lead ECG demonstrated $\mathrm{ST}$ elevation in V1-V3 with reciprocal changes (see Figure 2, C), and the patient was transferred to the angiography suite at 1314 hours on a norepinephrine infusion to help maintain blood pressure. He underwent percutaneous coronary angioplasty with stenting of two lesions in the left anterior descending (LAD) (99\% and $70 \%$ stenosis). An intra-aortic balloon pump was inserted at the time of angioplasty and removed within 
48 hours as his vasopressor requirements decreased. His maximum high-sensitivity troponin I was $>50000 \mathrm{ng} / \mathrm{ml}$.

During post-arresttargeted temperature management (target of $32^{\circ} \mathrm{C}-34^{\circ} \mathrm{C}$ ), the patient developed persistent hyperglycemia that was treated with insulin leading to severe hypokalemia $(1.5 \mathrm{mmol} / \mathrm{L})$, and another cardiac arrest that occurred approximately 24 hours after his hospital arrival. Standard ACLS care with traditional single defibrillator shocks and intravenous potassium were used with success. He later developed a ventilatorassociated pneumonia on post-admission Day 3 and episodes of delirium. He was discharged home on post-admission Day 14 neurologically intact with a cerebral performance category score of 1 .

\section{DISCUSSION}

\section{Background}

We present a case of successful resuscitation using DSED in the emergency department setting for RVF in an EMS-witnessed cardiac arrest secondary to ST-elevation myocardial infarction.

DSED has been previously reported to work for multiple etiologies of cardiac arrest. ${ }^{6,8,10-16}$ In one report of 2,990 consecutive patients undergoing treatment in the electrophysiology lab over a 3-year period, all 5 patients with VF refractory to standard defibrillation were successfully defibrillated with DSED. ${ }^{10}$

A handful of prehospital and emergency department case reports and small case series in recent years describe DSED. ${ }^{6,8,14-16}$ In one prehospital OHCA case series, with 10 patients, DSED converted RVF to an alternate rhythm with or without perfusion in 7 of 10 OHCA, although no patients survived to hospital discharge. ${ }^{6}$ A retrospective observational single centre study evaluating neurologically intact survival in OHCA patients with refractory or recurrent VF treated with DSED versus conventional therapy did not detect a statistically significant difference in the primary outcome of neurologically intact survival to hospital discharge. ${ }^{17}$ Three of 50 patients in the DSED group survived to discharge neurologically intact compared to 26 of 229 standard therapy patients. ${ }^{17}$ Important prognostic characteristics, such as bystander CPR and whether the arrest was witnessed, differed between the two groups, and there was no measure of the quality of CPR or record of when DSED was used during the cardiac arrest.
In the reports, authors suggest that DSED may have been used too late in the majority of resuscitations. Yet using DSED too soon makes it difficult to establish superior efficacy over conventional defibrillation because a subsequent shock may have been successful whether it was a DSED. In our case, DSED was attempted approximately 18 minutes after collapse and after four failed defibrillations with sustained ROSC achieved at 27 minutes.

\section{Proposed mechanisms}

Three mechanisms for DSED efficacy have been proposed: vector, total energy, and timing.

\section{Vector theory}

Vector has emerged as the most popular proposed mechanism for DSED. It is theorized that initial pad placement may not provide the correct energy vector, and thus a second shock from a second vector may be the key to success. ${ }^{6}$ Although external pad placement determines the overall vector of the energy discharge, anatomic variation and slight differences in pad placement may greatly change the current density traversing the ventricles. Gerstein has shown that delivery of current from multiple vectors is superior to a single vector when treating arrhythmias. ${ }^{13}$ It is theorized that shock vectors aligned with the ventricular septum have a higher success rate. ${ }^{13}$ Thus, two distinct and nearly orthogonal energy vectors increase the probability of optimal orientation to the interventricular septum. ${ }^{13,18}$ For example, Jones et al. have suggested that certain areas of myocardium receive lower current densities than others when an electrical pulse is passed between two poles (one set of pads), rather than when four poles are used (two sets of pads). ${ }^{19}$ See Figure 3 for a visual representation. Theoretically, repositioning pads when treating RVF using a single defibrillator may be as effective as DSED. On the basis of insufficient higherlevel evidence, ILCOR has yet to update its treatment recommendations on pad placement or energy level from the 2010 recommendations. ${ }^{20}$

\section{Timing theory}

Another proposed mechanism of DSED relates to the timing of the energy release. In DSED, the defibrillators discharge asynchronously, thereby prolonging the 

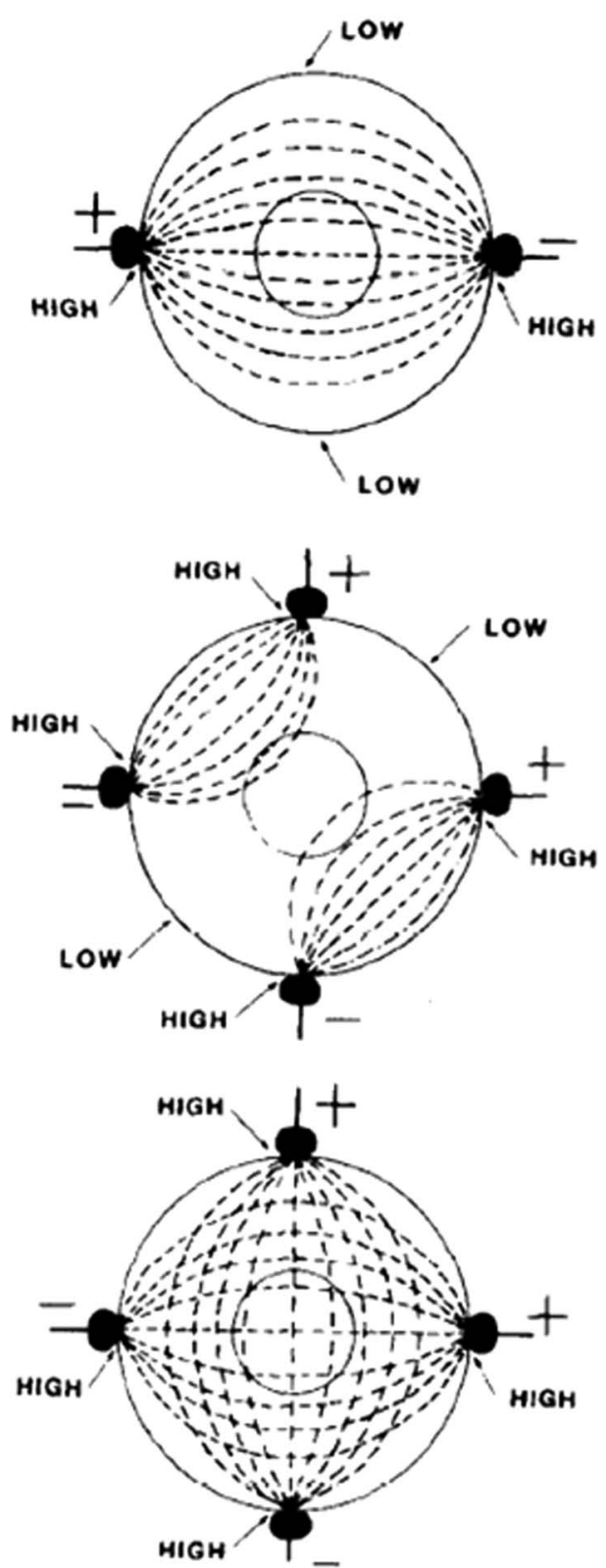

Figure 3 . Top: When a pulse is passed between two poles, there are regions with relatively high current density in the proximity of the electrodes and other regions with relatively low current density distant from the electrodes. To defibrillate myocardium, a sufficiently high amplitude pulse would have to be generated to depolarize a large portion of the myocardium. Bottom: There is a theoretical improvement in the distribution of current when four spatially located electrodes are used for countershock. However, if the two pulses are delivered simultaneously, there is not a configuration that does not lead to a "short circuit," as seen in the middle panel. With temporal and spatial separation, there is a closer approximation to the distribution located in the bottom panel.

(Reproduced with permission from Jones D, Klein G, Kallok M. Improved internal defibrillation with twin pulse sequential energy delivery to different lead orientations in pigs. Am J Cardiol 1985;55(6):821-5.) duration of defibrillation and the flow of current through the fibrillating myocardium. This is thought to increase the chance of successful defibrillation. ${ }^{13,14}$ Human studies have shown that multiple sequential shocks decrease the threshold for defibrillation. ${ }^{21}$ Using canines, Johnson et al. varied the time between shocks in DSED to determine the optimal timing separation of shocks. ${ }^{22}$ Shocks in rapid succession of either $<10 \mathrm{msec}$ or $>75 \mathrm{msec}$ and $<125 \mathrm{msec}$ have a higher chance of success. $^{22}$ In contrast, shocks around the refractory period at approximately $50 \mathrm{msec}$ have a lower probability of success, requiring a substantially higher voltage to convert VF into a perfusing rhythm. ${ }^{22}$ Johnson et al. also found that two shocks separated by the $75-125$ msec period, successfully defibrillated dogs at an energy level that would have failed to defibrillate the myocardium after a single shock. ${ }^{22}$ The current, somewhat naïve practice of attempting to synchronize two shocks using separate defibrillators leads to the shocks being delivered at slightly different times. Ensuring that the interval between the shocks actually falls within the optimal time range that would maximize the chance for successful defibrillation will require modification of the existing technology.

\section{Total energy theory}

Finally, the total energy delivered to the myocardium may contribute to the success of DSED. A second defibrillator discharging nearly simultaneously with the first may increase the intracardiac voltage gradient above the threshold required to terminate VF. Zhang et al. suggest that body habitus has a large role to play in external defibrillation success. ${ }^{23}$ In a swine model, this group demonstrated that a larger BMI would require a higher biphasic energy level to obtain the appropriate current to eliminate VF. ${ }^{23}$ Energy levels of up to $720 \mathrm{~J}$ $(360 \mathrm{~J} \times 2)$ have been used for DSED; this total energy delivered may be partly responsible for its success, especially in larger patients. ${ }^{10}$

\section{CONCLUSION}

Recent reports of anecdotal success with DSED support the biologic rationale, feasibility, and potential efficacy of DSED for treatment of RVF. DSED should be evaluated with a well-designed randomized controlled trial to determine the optimal configuration of electrical defibrillation for VF. 
Acknowledgement: The authors would like to thank Dr. Marco Sivilotti for his help with proofreading this manuscript.

Competing interests: None declared.

\section{REFERENCES}

1. Nichol G, Thomas E, Callaway C, et al.. Regional variation in out-of-hospital cardiac arrest incidence and outcome. 7AMA 2008;300(12):1423-31.

2. Heart and Stroke Foundation of Canada. Statistics; 2016. Available at: http://www.heartandstroke.com/site/c.ikIQLc MWJtE/b.3483991/k.34A8/Statistics.htm (accessed 27 July 2016).

3. Link M, Berkow L, Kudenchuk P, et al. Part 7: adult advanced cardiovascular life support. Circulation 2015; 132(18 Suppl 2):S444-64.

4. Kudenchuk P, Brown S, Daya M, et al. Amiodarone, lidocaine, or placebo in out-of-hospital cardiac arrest. $N$ Engl $\mathcal{Z}$ Med 2016;375(8):801-3.

5. Sakai T, Iwami T, Tasaki O, et al. Incidence and outcomes of out-of-hospital cardiac arrest with shock-resistant ventricular fibrillation: data from a large populationbased cohort. Resuscitation 2010;81(8):956-61.

6. Cabañas J, Myers J, Williams J, et al. Double sequential external defibrillation in out-of-hospital refractory ventricular fibrillation: a report of ten cases. Prehosp Emerg Care 2014;19(1):126-30.

7. Sarkozy A, Dorian P. Strategies for reversing shock-resistant ventricular fibrillation. Curr Opin Crit Care 2003;9(3):189-93.

8. Leacock B. Double simultaneous defibrillators for refractory ventricular fibrillation. 7 Emerg Med 2014;46(4):472-4.

9. Lee $\mathrm{YH}$, Lee KJ, Min YH, et al. Refractory ventricular fibrillation treated with esmolol. Resuscitation 2016;107:150-5.

10. Hoch D, Batsford W, Greenberg S, et al. Double sequential external shocks for refractory ventricular fibrillation. $7 \mathrm{Am}$ Coll Cardiol 1994;23(5):1141-5.

11. Marrouche N, Bardy G, Frielitz H, et al. Quadruple pads approach for external cardioversion of atrial fibrillation. PACE 2001;24(9):1321-4.

12. Alaeddini J, Feng Z, Feghali G, et al. Repeated dual external direct cardioversions using two simultaneous 360-J shocks for refractory atrial fibrillation are safe and effective. PACE 2005;28(1):3-7.

13. Gerstein N, Shah M, Jorgensen K. Simultaneous use of two defibrillators for the conversion of refractory ventricular fibrillation. 7 Cardiothorac Vasc Anesth 2015;29 (2):421-4.

14. Lybeck A, Moy H, Tan D. Double sequential defibrillation for refractory ventricular fibrillation: a case report. Prehosp Emerg Care 2015;19(4):554-7.

15. Merlin M, Tagore A, Bauter R, et al. A case series of double sequence defibrillation. Prehosp Emerg Care 2016; 20(4):550-3.

16. Johnston M, Cheskes S, Ross G, et al. Double sequential external defibrillation and survival from out-of-hospital cardiac arrest: a case report. Prehosp Emerg Care 2016; 20(5):662-6.

17. Ross E, Redman T, Harper S, et al. Dual defibrillation in cardiac arrest: a retrospective cohort analysis. Resuscitation 2016;106:14-7.

18. Kerber R, Spencer K, Kallok M, et al. Overlapping sequential pulses. A new waveform for transthoracic defibrillation. Circulation 1994;89(5):2369-79.

19. Jones D, Klein G, Kallok M. Improved internal defibrillation with twin pulse sequential energy delivery to different lead orientations in pigs. Am $\mathcal{F}$ Cardiol 1985; 55(6):821-5.

20. Morrison L, Deakin C, Morley P, et al. Part 8: advanced life support: 2010 international consensus on cardiopulmonary resuscitation and emergency cardiovascular care science with treatment recommendations. Circulation 2010; 122(16 Suppl 2):S345-421.

21. Jones D, Klein G, Guiraudon G, et al. Internal cardiac defibrillation in man: pronounced improvement with sequential pulse delivery to two different lead orientations. Circulation 1986;73(3):484-91.

22. Johnson E, Alferness C, Wolf $\mathrm{P}$, et al.. Effect of pulse separation between two sequential biphasic shocks given over different lead configurations on ventricular defibrillation efficacy. Circulation 1992;85(6): 2267-74.

23. Zhang Y, Clark C, Davies L, et al. Body weight is a predictor of biphasic shock success for low energy transthoracic defibrillation. Resuscitation 2002;54(3):281-7. 